ORIGINAL ARTICLE

\title{
Sleep apnoea attenuates the effects of a lifestyle intervention programme in men with visceral obesity
}

\author{
Anne-Laure Borel, ${ }^{1}$ Xavier Leblanc, ${ }^{2}$ Natalie Alméras, ${ }^{1}$ Angelo Tremblay, ${ }^{1,3}$ \\ Jean Bergeron, ${ }^{4}$ Paul Poirier, ${ }^{1}$ Jean-Pierre Després, ${ }^{1,3}$ Frédéric Series ${ }^{1}$
}

\begin{abstract}
- Additional materials are published online only. To view these files please visit the journal online (http://thorax.bmj. com/content/67/8.toc).

${ }^{1}$ Centre de recherche, Institut universitaire de cardiologie et de pneumologie de Québec, Québec, Canada

${ }^{2}$ Faculty of Medicine, Université Laval, Québec, Canada ${ }^{3}$ Department of Social and Preventive Medicine, Division of Kinesiology, Université Laval, Québec, Canada

${ }^{4}$ Lipid Research Center, CHUO Research Center, Québec Canada
\end{abstract}

\section{Correspondence to} Dr Frédéric Sériès, Department of Pneumology, Institut universitaire de cardiologie et de pneumologie de Québec, 2725 Chemin Sainte-Foy, G1V 4 G5 Québec, Canada;

frederic.series@fmed.ulaval.ca

Received 26 August 2011 Accepted 14 February 2012 Published Online First 6 March 2012
ABSTRACT

Background Excess visceral adiposity and sleep apnoea are two conditions independently associated with cardiovascular diseases. The two conditions are often combined and are believed to interact in a vicious circle. Objectives To compare the response of men with visceral obesity with or without sleep apnoea syndrome to a 1-year healthy eating, physical activity/exercise intervention programme.

Methods 77 men, selected on the basis of increased waist circumference $(\geq 90 \mathrm{~cm})$ and dyslipidaemia (triglycerides $\geq 1.69$ and/or high-density lipoprotein (HDL) cholesterol $<1.03 \mathrm{mmo} /$ /itre), participated in this study. Body composition and fat distribution were assessed by dual-emission X-ray absorptiometry or CT and sleep breathing disorders by home-based polygraphic recording. Cardiorespiratory fitness, plasma adipokines, plasma inflammatory markers, fasting lipoprotein-lipid profile and oral glucose tolerance test were assessed.

Results After the 1-year lifestyle intervention, the mean oxygen desaturation index (ODI) of the whole sample decreased $(-3 \pm 13$ events/h, $p<0.05)$. Men with sleep apnoea syndrome at baseline (ODI $\geq 10$ events/h, $n=28$ ) showed smaller reductions in body mass index, waist circumference, triglycerides and smaller increases in HDL cholesterol and adiponectin than men without sleep apnoea (ODI $<10$ events/h, $n=49$ ), despite similar compliance to the programme. The higher the baseline $\mathrm{ODI}$ and the time spent under $90 \%$ saturation, the lower the reductions in fat mass and visceral adiposity, and the smaller the improvement in glucose/insulin homeostasis indices after 1 year.

Conclusions Men with sleep apnoea syndrome at baseline had smaller reduction in body weight and less metabolic improvements associated with the lifestyle intervention programme than men without sleep apnoea syndrome.

\section{INTRODUCTION}

Excess visceral adipose tissue (VAT) and obstructive sleep apnoea syndrome (OSAS) are two conditions independently associated with an increased risk of cardiovascular diseases and mortality. ${ }^{1} 2$ Both conditions are linked with increased insulin resistance, inflammatory cytokines and blood pressure levels. ${ }^{3-6}$ Moreover, excess VAT and OSAS are often associated conditions which are believed to interact with each other in a vicious circle. ${ }^{67}$ For instance, Peppard et al $l^{8}$ demonstrated that $10 \%$ weight gain

\section{Key messages}

What is the key question?

- Is the response similar between men with visceral obesity with or without sleep apnoea syndrome to a 1-year healthy eating, physical activity/exercise intervention programme?

What is the bottom line?

- Excess visceral adiposity and sleep apnoea are two conditions independently associated with an increased risk of cardiovascular diseases. The two conditions are often combined and are believed to favour a reciprocal worsening.

\section{Why read on?}

- Sleep-induced disordered breathing of men with visceral obesity was improved after a 1-year healthy eating, physical activity/exercise programme. The presence of sleep-disordered breathing at baseline attenuated the reduction of body weight and the improvement of the cardiometabolic risk profile in response to the 1 -year lifestyle intervention.

or weight loss were respectively associated with a $32 \%$ increase and a $26 \%$ decrease in the apnoea + hypopnoea index (AHI), whereas the level of physical activity was inversely associated with the level of AHI. ${ }^{9}$ Of importance, the treatment of OSAS by continuous positive airway pressure (CPAP) has been associated with a VAT loss, independent of the change in body weight. ${ }^{10}$

Three recent studies have tested the efficacy of lifestyle intervention programmes to reduce AHI in patients with OSAS. ${ }^{11-13}$ All these studies found that the body weight loss related to lifestyle interventions was associated with a decrease in AHI. These results were sustained after 1 year of follow-up. ${ }^{14}{ }^{15}$ However, whether the benefit of such lifestyle intervention programmes to improve the cardiometabolic risk profile could be affected by the presence and severity of sleep-disordered breathing has not been addressed.

We recently investigated a group of men with visceral obesity, defined as abdominal obesity and the dyslipidaemia of insulin resistance ${ }^{13}$ that is, high triglyceride and/or low high-density lipoprotein (HDL) cholesterol levels, who were involved in a healthy eating, physical activity/exercise lifestyle modification programme. In this study, we 
addressed the presence and severity of sleep breathing disorders in an ancillary study conducted to compare the response of men with visceral obesity with or without OSAS to a 1-year lifestyle intervention; that is, to test whether having sleep-disordered breathing could impede the improvement of the cardiometabolic risk profile in response to a 1-year healthy eating, physical activity/exercise lifestyle modification programme.

\section{METHODS}

Further information on the methods is presented in an online supplementary file.

\section{Study design}

Men $(n=144)$ between 30 and 65 years old, presenting with a waist circumference $\geq 90 \mathrm{~cm}$, triglyceride levels $\geq 1.69 \mathrm{mmol} /$ litre and/or HDL cholesterol $<1.03 \mathrm{mmol} /$ litre were recruited in the general community to take part in a 3-year lifestyle modification programme (the 'SYNERGIE' study, to emphasise the synergism between healthy eating and increased physical activity/exercise). From these 144 men, 86 volunteered to participate in an ancillary study addressing nocturnal respiratory disturbances at baseline and after 1 year. From these 86 men, 77 had a technically acceptable sleep home-recording, and were kept for baseline analyses, whereas data on 66 men were available for the 1-year analyses (figure 1). Written consent was obtained for every study participant which was approved by the Medical Ethics Committees of Universite Laval and of the Institut universitaire de cardiologie et de pneumologie de Québec.

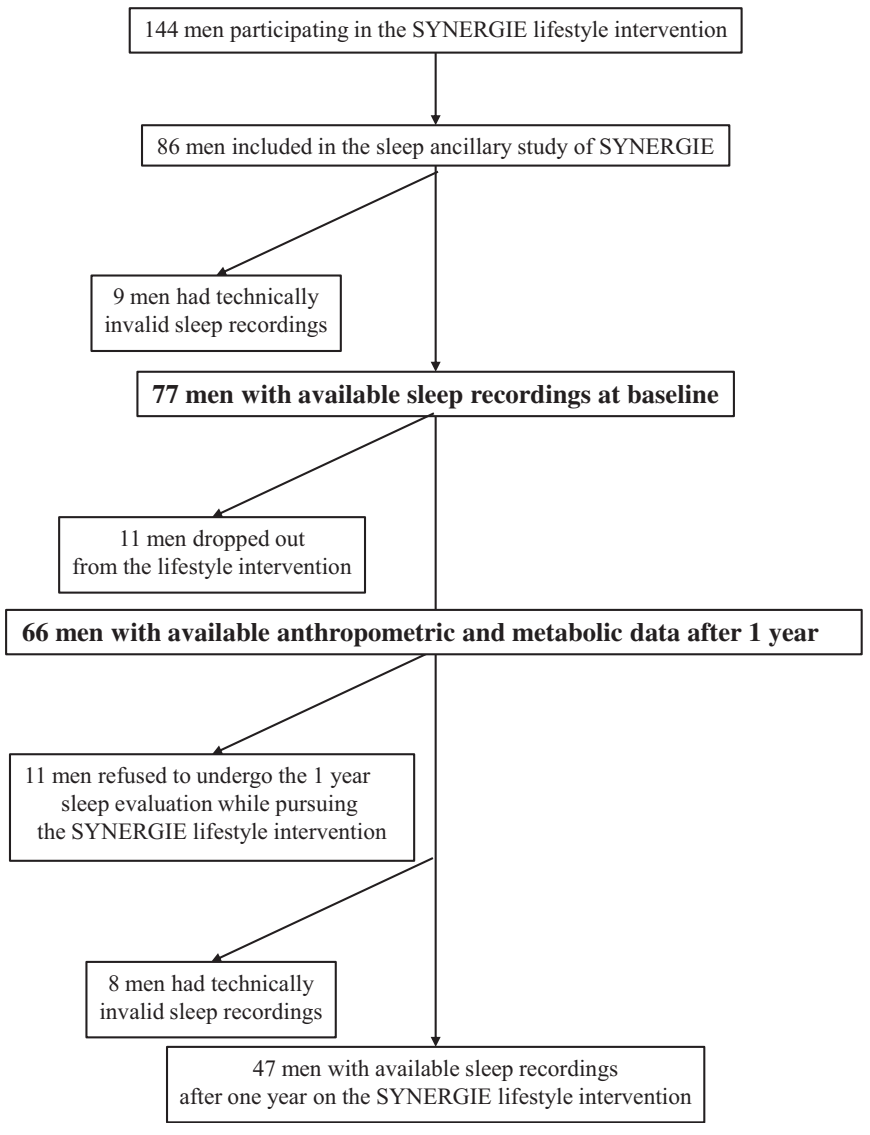

Figure 1 Flow chart.
Subjects were individually counselled once every 2 weeks during the first 4 months of management with subsequent monthly visits to improve their nutritional and physical activity/exercise habits. Each visit included an interactive session with a registered nutritionist followed by a meeting with a kinesiologist. The nutritional counselling was adapted to elicit a $500 \mathrm{kcal}$ daily energy deficit during the first year, which was the 'moderate weight loss' phase of SYNERGIE. The daily caloric intake was estimated at baseline and at 1 year by a 3-day dietary record including a non-working day. The physical activity programme aimed to reach $160 \mathrm{~min} /$ week of moderate intensity endurance exercise. Men received a personalised training programme elaborated according to subjects' history and preferences and based on results to a maximal treadmill test and on the rating of self-perceived exhaustion (modified Borg scale $^{16}$ ). Men had free access to an on-site fitness centre and were allowed to perform exercise on site or outside at their convenience. To help participants to be more active and to monitor themselves between exercise sessions, they were asked to wear a pedometer and to reach a target of 10000 daily steps.

\section{CT scan}

Partial volumes of VAT and subcutaneous abdominal adipose tissue were calculated ${ }^{17}$ from cross-sectional areas assessed at L2-L3 and L4-L5 by CT. ${ }^{18} 19$

\section{Cardiorespiratory fitness}

A submaximal treadmill standardised exercise test evaluated cardiorespiratory fitness using two indices: the subject's heart rate (mean of the last $3 \mathrm{~min}$ ) at a submaximal treadmill work-

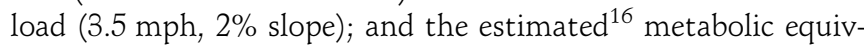
alent of task reached by the subject at a heart rate of 150 beats/ $\min$.

\section{Oral glucose tolerance test}

Participants were subjected to a standard, $3 \mathrm{~h}, 75 \mathrm{~g}$ oral glucose tolerance test (OGTT).

\section{Nocturnal respiratory recording}

Daytime sleepiness was evaluated with the Epworth Sleepiness Scale. $^{20}$ Presence of nocturnal respiratory disturbances was assessed at home (Remmers Sleep Recorder Model 4.2; Saga Tech Electronic, Calgary, Alberta, Canada). The oxygen desaturation index (ODI) (number of oxygen saturation $\left(\mathrm{SpO}_{2}\right)$ decreases $\geq 4 \%$ /recording time), mean $\mathrm{SpO}_{2}$, minimal $\mathrm{SpO}_{2}$, time spent under $90 \%$ of $\mathrm{SpO}_{2}$ and total monitoring time were automatically determined. ${ }^{21}$ Apnoea and hypopnea lasting $\geq 10 \mathrm{~s}$ were scored to determine the respiratory disturbance index (RDI). ODI was used to establish sleep apnoea phenotype using the $10 / \mathrm{h}$ ODI cut-off to separate participants with or without moderate to severe OSAS, as previously described. ${ }^{21} 22$

\section{Statistical analysis}

The primary outcome of the SYNERGIE study was the VAT volume change after the intervention. In the present ancillary study, the primary outcome was the change in ODI after the 1year lifestyle intervention. Baseline and 1-year results were expressed as mean (SD) for normally distributed variables and as median (IOR) for non-normally distributed variables. One year changes are expressed as mean (95\% CI).

Statistical significance of changes after 1 year was evaluated using mixed linear models with the time of measurement (baseline and 1 year) as a repeated measure on the 77 patients who completed the sleep recording at baseline (table 1). 
Table 1 Characteristics of men with visceral obesity at baseline and after 1 year on the lifestyle intervention programme

\begin{tabular}{|c|c|c|c|c|}
\hline & Baseline $(n=77)$ & 1 year $(n=66)$ & 1-year change $(n=66)$ & p Value \\
\hline Age (years) & $49.3(8.1)$ & $51.0(7.4)$ & $+1.1(+1.1$ to +1.1$)$ & \\
\hline BMI $\left(\mathrm{kg} / \mathrm{m}^{2}\right)$ & $30.6(3.1)$ & $28.7(3.3)$ & $-2.3(-2.6$ to -1.9$)$ & $\mathrm{p}<0.001$ \\
\hline Waist circumference $(\mathrm{cm})$ & $107.8(8.8)$ & $99.7(10.1)$ & $-8.6(-9.9$ to -7.4$)$ & $\mathrm{p}<0.001$ \\
\hline Diastolic blood pressure $(\mathrm{mm} \mathrm{Hg})$ & $82(7)$ & $76(7)$ & $-6(-7$ to -4$)$ & $\mathrm{p}<0.001$ \\
\hline Heart rate (beats/min) & $69(8)$ & $62(8)$ & $-7(-9$ to -4$)$ & $\mathrm{p}<0.001$ \\
\hline \multicolumn{5}{|l|}{ CT: L4-L5 area of adipose tissue } \\
\hline \multicolumn{5}{|l|}{ DEXA body composition } \\
\hline Fat-free mass $(\mathrm{kg})$ & $64(7)$ & $64(6)$ & $-1(-1$ to -0$)$ & $\mathrm{p}=0.002$ \\
\hline Fat mass $(\mathrm{kg})$ & $29(7)$ & $23(8)$ & $-6(-7$ to -5$)$ & $\mathrm{p}<0.001$ \\
\hline \multicolumn{5}{|l|}{ Submaximal treadmill exercise } \\
\hline Heart rate $-3.5 \mathrm{mph} ; 2 \%$ (beats $/ \mathrm{min}$ ) & $116(13)$ & $104(12)$ & $-13(-15$ to -10$)$ & $\mathrm{p}<0.001$ \\
\hline Minimum $\mathrm{SpO}_{2}(\%)$ & $84(6)$ & $82(8)$ & $-1(-2$ to +1$)$ & $\mathrm{p}=0.311$ \\
\hline Time spent under $90 \% \mathrm{SpO}_{2}(\% \mathrm{TRT})$ & $0.4(0.2-2.4)$ & $0.5(0.1-3.1)$ & $-0.9(-2.2$ to 0.5$)$ & $\mathrm{p}=0.217$ \\
\hline ODI (events/h) & $7.5(4.4-13.7)$ & $5.8(3.8-13.7)$ & $-2.7(-6.6$ to 1.1$)$ & $\mathrm{p}=0.025$ \\
\hline RDI (events/h) & $17.2(12.0-27.3)$ & $8.0(4.7-11.4)$ & $-11.3(-17.2$ to -2.3$)$ & $\mathrm{p}<0.001$ \\
\hline Total recording time (min) & 437 (88) & $414(105)$ & $-18(-47$ to 10$)$ & $\mathrm{p}=0.128$ \\
\hline
\end{tabular}

Data are presented as mean (SD) for baseline and 1-year values or median (IOR) for non-normally distributed values. Data are presented as mean (95\% CI) for 1-year change. P values report results of analysis of variance repeated measures.

BMI, body mass index; DEXA, dual-emission X-ray absorptiometry; METs, metabolic equivalents of task; ODI, oxygen desaturation index; RDI, respiratory disturbance index; SAT, subcutaneous abdominal adipose tissue; $\mathrm{SpO}_{2}$, oxygen saturation; TRT, total recording time; VAT, visceral adipose tissue.

Men were then classified according to baseline ODI value, below or above 10 events/h. Comparisons between the two groups at baseline were made by mixed linear model. The two groups were also compared for the 1-year changes with the same mixed linear model. One-year changes were then adjusted for baseline VAT volume and baseline daily step count and compared with analysis of covariance models (table 2).

Linear regressions were quantified between baseline ODI (log transformed) with 1-year changes of cardiometabolic risk markers. Spearman rank correlations were computed between baseline time spent under $90 \% \mathrm{SpO}_{2}$ and 1-year changes of cardiometabolic risk markers. ODI, RDI, time spent under $90 \%$ of $\mathrm{SpO}_{2}$, triglycerides, homeostatic model assessment-insulin resistance, C-reactive protein, tumour necrosis factor $\alpha$ (TNF $\alpha$ ) and interleukin- 6 values were log transformed because of skewed distribution, and all analyses involving these data used log transformed data. Significance was set at $p$ values $<0.05$. Analyses were carried out using SAS, V.9.2.

\section{RESULTS}

Eighty-six per cent of men with visceral obesity involved in this ancillary study completed the 1-year intervention. The 11 men who dropped out of the lifestyle intervention did not differ at baseline from the 66 men who completed the programme, except for body mass index (BMI) (28.9 $\pm 2.1 \mathrm{vs}$ $30.9 \pm 3.2$ in men who dropped out vs men who did not, $\mathrm{p}=0.04)$, TNF $\alpha(1.47 \pm 0.74$ vs $1.02 \pm 0.47, \mathrm{p}=0.01)$ and daily caloric intake $(2608 \pm 538$ vs. $3069 \pm 579, p=0.02)$. The 19 men with visceral obesity who completed the 1-year lifestyle intervention but did not have the 1-year sleep evaluation presented with lower heart rate $(65 \pm 7$ vs $70 \pm 8$ for men who did not have the 1-year sleep evaluation compared with the others, $p=0.05)$, triglycerides $(2.20 \pm 1.02$ vs $2.54 \pm 0.81$, $\mathrm{p}=0.03)$ and fasting plasma insulin $(151 \pm 89$ vs $174 \pm 78$, $p=0.03$ ) than other men at baseline. However, they were no different from the others after 1 year of intervention, except for fasting insulin which remained lower $(100 \pm 60$ vs $109 \pm 37$, $\mathrm{p}=0.04$ ).

\section{One-year response to the lifestyle modification programme and changes in sleep-disordered breathing variables}

Baseline characteristics of men with visceral obesity and their 1year responses to the lifestyle intervention programme are reported in table 1. After 1-year of lifestyle intervention, they had lost $26.7 \%$ of VAT and had improved their cardiorespiratory fitness by $21.3 \%$. When considering absolute changes, Epworth score, time spent under $90 \%$ of $\mathrm{SpO}_{2}, \mathrm{ODI}$ and $\mathrm{RDI}$ all decreased after 1 year of lifestyle intervention, whereas mean $\mathrm{SpO}_{2}$ increased.

\section{Results of the lifestyle intervention in men with visceral obesity with baseline ODI below or above 10 events/h}

To assess whether nocturnal oxygen desaturations could alter the response to a 1-year healthy eating physical activity/exercise lifestyle intervention, men with visceral obesity were classified into two groups according to their baseline ODI with a 10 events/h cut-off. Twenty-eight out of 77 men presented an ODI above 10 events/h at baseline (36\%).

At baseline, men with nocturnal oxygen desaturations above 10 events/h (ODI $24 \pm 18$ events/h) presented with a lower mean $\mathrm{SpO}_{2}(93 \pm 3$ vs $95 \pm 1 \%$ for men with baseline ODI above vs below 10 events $/ \mathrm{h}$ at baseline, respectively, $\mathrm{p}<0.001)$ and minimum $\mathrm{SpO}_{2}(80 \pm 8$ vs $86 \pm 3 \%)$ than those with less frequent nocturnal oxygen desaturations (ODI $5 \pm 2$ events/h), whereas they presented with higher RDI ( $32 \pm 21$ vs $13 \pm 5$ events/h) and time spent under $90 \%$ of $\mathrm{SpO}_{2}$ (8 \pm 13 vs $1 \pm 1 \%$ of sleep recorded time). There were no differences in Epworth scores. There were no differences at baseline between the two groups for 
Table 2 Response to the 1-year healthy eating, physical activity/exercise lifestyle modification programme according to oxygen desaturation index level at baseline



$\mathrm{p}$ values in bold indicate significant difference.

Data are presented as mean (SD) for baseline values or median (IOR) for non-normally distributed values. Data are presented as mean (95\% $\mathrm{CI}$ ) for 1-year change.

Comparisons were tested between the two groups. Comparisons of the 1-year changes have been made unadjusted and adjusted for baseline visceral adipose tissue and baseline daily step count. AUC, area under the curve; BMI, body mass index; CRP, C-reactive protein; DEXA, dual-emission X-ray absorptiometry; HDL, high-density lipoprotein; HOMA-IR, homeostatic model assessment-insulin resistance; IL-6, interleukin-6; METs, metabolic equivalents of task; ODI, oxygen desaturation index; OGTT, oral glucose tolerance test; SAT, subcutaneous abdominal adipose tissue; TNF $\alpha$, tumour necrosis factor $\alpha$; VAT, visceral adipose tissue.

anthropometric and metabolic variables, except for a higher area under the curve for glucose and a lower daily step count in those with baseline ODI above 10 events/h (table 2).

After 1 year of intervention, men with visceral obesity and a baseline ODI above 10 events/h showed smaller decreases in BMI, waist circumference, fat mass and triglyceride, and smaller increases in adiponectin and HDL cholesterol levels than men with baseline ODI below 10 events/h. Changes in daily step counts and in reported daily caloric intake after 1 year of intervention did not differ between the two groups.

When these comparisons were adjusted for baseline VAT volume and baseline daily step counts, the differences between subgroups remained for the 1-year changes in BMI, waist circumference, triglycerides and fat mass, whereas differences in 1-year changes in HDL cholesterol and adiponectin levels were no longer significant.

\section{Associations between baseline sleep characteristics and changes in anthropometric and cardiometabolic risk markers} Finally, linear regression was quantified between baseline ODI (log transformed) and 1-year changes of cardiometabolic risk markers. Reductions in fat mass and 120 min OGTT were less substantial after 1 year of lifestyle intervention when baseline ODI was high (figure 2). Furthermore, Spearman rank correlation was analysed between baseline time spent under $90 \% \mathrm{SpO}_{2}$ and 1-year changes in cardiometabolic risk markers: the longer the time spent under $90 \% \mathrm{SpO}_{2}$ at baseline, the lower the reductions in $120 \mathrm{~min}$ OGTT and VAT. 
Figure 2 Association between baseline sleep variables and 1-year changes in body composition/fat distribution, plasma glucose/insulin homeostasis. Linear regressions (left) were searched between baseline oxygen desaturation index (log transformed, logODI) and the 1-year changes in fat mass, visceral adipose tissue (VAT) and 120 min oral glucose tolerance test (OGTT)-glucose. Spearman rank correlations (right) were searched between time spent under $90 \%$ oxygen saturation $\left(\mathrm{SaO}_{2}\right)$ at baseline and the 1-year changes in fat mass, visceral adipose tissue and 120 min OGTT-glucose.
LogODI at baseline

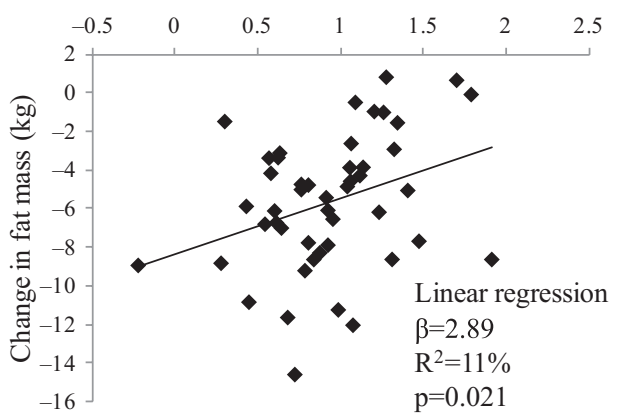

Time spent under $90 \%$ of $\mathrm{SaO}_{2}$ at baseline (\%)
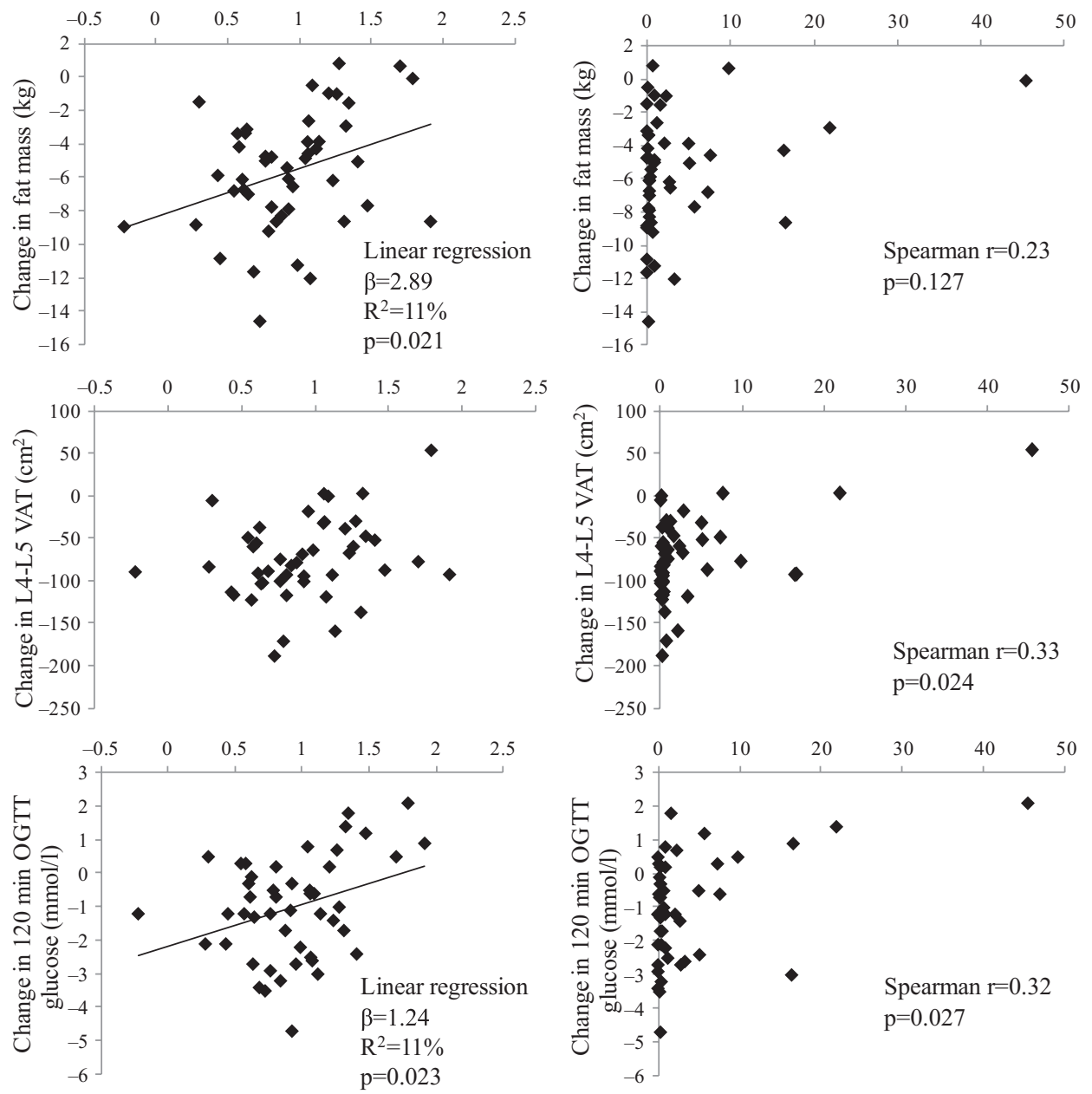

\section{DISCUSSION}

This study was conducted to assess whether having a sleep apnoea syndrome (defined by a baseline ODI above 10 events/h) at baseline could impede the response to the 1-year lifestyle intervention. Men with visceral obesity and a baseline ODI above 10 events/h presented with a lower decrease in BMI, waist circumference and fat mass after 1-year of intervention than men with a baseline ODI below 10 events/h, in spite of a similar compliance to the programme. Accordingly, these men presented smaller improvements in their cardiometabolic risk markers after 1 year. This study was not designed as a randomised trial to evaluate the effects of a lifestyle modification programme on nocturnal respiratory disturbances. Indeed, the efficacy of lifestyle modification programmes to improve nocturnal respiratory disturbances has already been well documented, ${ }^{11-15}{ }^{23}$ and as expected, this 1-year lifestyle intervention was efficient in improving the cardiometabolic risk profile and nocturnal respiratory disturbances in these men. The dropout rate was $14 \%$ for participation in the lifestyle intervention programme and 19 men had invalid data or refused to undergo the home sleeprecording procedure at 1 year. These missing data are the consequence of the great burden associated with our extensive testing protocol in the SYNERGIE study. Thus, ancillary tests were more often turned down by participants than the tests planned in the main protocol. This is also why sleep respiratory disturbances were assessed by home-recording polygraphy rather than with polysomnography at the sleep clinic.
Efficiency of the lifestyle intervention programme in improving sleep-disordered breathing

There is strong evidence that body weight excess is a causal factor in OSAS. ${ }^{24}$ While most studies addressing the effect of weight loss on AHI have examined the effects of surgically induced body weight loss, ${ }^{7} 23$ data regarding the feasibility of a non-surgical body weight loss programme as a means to improve OSAS have also been reported. ${ }^{11} 1225$ Recently, three randomised clinical trials have tested the efficiency of lifestyle intervention to improve sleep-disordered breathing. Tuomilehto et $a l^{11}$ have shown that in patients with mild OSAS (AHI 9.3 events/h at baseline), a lifestyle intervention programme based on a very low caloric diet for 3 months with subsequent dietary counselling for 1 year was efficient to induce significant decreases in body weight, waist circumference and $\mathrm{AHI}$ compared with patients receiving only non-specific information. Body weight and waist circumference losses were strongly related to reduction in AHI during the 12-month follow-up period, with a linear dose-response. In the Tuomilehto study, the mean decrease in AHI was 4 events/h in patients who lost 5-10 kg after 1 year of intervention. In the present study, we observed a decrement of 3 events/h in ODI in response to the same range of body weight loss. Foster et al ${ }^{12}$ conducted an ancillary study of the look AHEAD study, which included a polysomnographic study at baseline and after 1 year. Adults who were overweight or obese and had type 2 diabetes were randomised to intensive diet and exercise lifestyle intervention 
or to diabetes support and education. Patients in the first group lost more weight and had a greater decrease in AHI than patients assigned to the conventional support and education group. Baseline AHI was higher in patients enrolled in the look AHEAD ancillary study (22.9 events/h) than in those enrolled in the Tuomilehto study. Johansson et al ${ }^{13}$ conducted a 9 -week very low caloric diet intervention consisting of liquid meals for 7 weeks followed by 2 weeks of progressive return to normal food in men with mild to severe OSAS (baseline AHI 37 events/h). Subjects also received counselling to help them increase their physical activity. Subjects of the intervention arm lost a mean of $20 \mathrm{~kg}$ in 9 weeks with a decrease in AHI of 23 events/h compared with subjects in the control arm. Following a 1-year weight maintenance programme, the mean body weight loss was $12 \mathrm{~kg}$ and the decrease of AHI was 17 events/h compared with baseline. ${ }^{15}$ In this study, the range of baseline ODI values was large, from 0.6 to 82.0 events/h. Our finding that nocturnal breathing disturbance indices could be improved by a healthy eating and physical activity/exercise programme in men with visceral obesity is in accordance with the results of the abovementioned diet and exercise lifestyle intervention studies conducted in subjects with mild to severe OSA and in those who are overweight or obese and have type 2 diabetes.

\section{Why OSAS impaired the response to the lifestyle intervention}

Current opinion is that OSAS is a condition associated with a particular difficulty to lose weight and may even have a causal role in weight gain. ${ }^{7}$ Nevertheless, such an assumption is not based on robust evidence from the literature. Phillips et al ${ }^{26}$ showed that weight gain in the year preceding the diagnosis of OSAS was significantly higher than that observed in matched subjects free of OSAS, whereas Traviss et $a^{27}$ found that $84 \%$ of patients having OSAS self-reported weight gain following the onset of symptoms. Both studies assessed body weight changes based on self-reported, retrospective data. However, a recent randomised controlled crossover study published by Sharma et $a^{28}$ found that CPAP therapy improves features of the metabolic syndrome and body weight and VAT in patients with mild to severe OSAS, suggesting that OSAS has a deleterious independent effect on these parameters. This study found new evidence that despite similar compliance to the healthy eating, physical activity/exercise programme (the sleep apnoea status did not influence the decrease in daily caloric intake or the increase in daily step counts after 1 year), subjects having a baseline ODI above 10 events/h showed a smaller reduction in body weight, waist circumference and fat mass, and had less substantial improvements in cardiometabolic risk markers than those with a baseline ODI below 10 events/h.

Although further studies are needed to confirm these findings, OSAS-related resistance to weight loss strategies may be associated with alterations in sleep duration and/or continuity. ${ }^{29-31}$ Indeed, OSAS is characterised by recurrent episodes of partial or complete upper airways collapse occurring during sleep, which lead to sleep fragmentation, nocturnal oxygen desaturation and shorter total sleep time. Evidence from epidemiological studies suggests that short sleep duration is associated with weight gain. ${ }^{32} 33$ This observation has been reinforced by mechanistic studies evaluating the effect of sleep restriction on appetite and metabolism. These works have shown that sleep restriction is associated with increased appetite, increased ghrelin and decreased leptin levels. ${ }^{30} 31$ Beside the effect of sleep restriction by itself, some studies have shown that sleep fragmentation is associated with a decrease in insulin sensitivity and glucose utilisation in relation to increased sympathetic activity and cortisol levels. ${ }^{34} 35$ Indeed, OSAS is associated with an activation of the hypothalamic-pituitary-adrenal axis in response to stress caused by recurrent intermittent hypoxia, sleep fragmentation and frequent cerebral arousals during apnoeic events. This activation, which is corrected by CPAP treatment, ${ }^{36}$ could explain body weight gain or difficulty to achieve body weight loss in patients having OSAS. Finally, some studies suggest that patients characterised by a pronounced recurrent transient oxygen desaturation profile have a smaller energy expenditure relative to their body weight. ${ }^{37}$ This phenomenon could eventually be explained by the presence of a decrease in thermogenesis associated with an increase in oxygen desaturation severity of OSAS. ${ }^{38}$ However, this observation is controversial since one other controlled study found an increase in 24 h energy expenditure in patients with OSAS. ${ }^{39}$

The findings from these studies suggest why OSAS could affect body weight loss and improvement in cardiometabolic risk variables in people on a lifestyle intervention programme. However, further studies are needed to examine whether the effects demonstrated by sleep restriction and sleep fragmentation studies on energy metabolism are extendable to patients presenting with the intricate pathophysiology of OSAS. In light of these results, whether sleep apnoea treatment would improve the efficiency of a lifestyle intervention programme in patients with OSAS should also be examined. Kajaste et $a^{25}$ conducted a 2-year cognitive-behavioural weight loss programme in patients with obesity $\left(\mathrm{BMI}>35 \mathrm{~kg} / \mathrm{m}^{2}\right)$ and OSAS. They randomised 16 and 15 subjects into two groups with or without concomitant CPAP therapy during the first 6 months. No difference in body weight loss was found between the two groups at 6 months or thereafter, although compliance to CPAP treatment was not controlled. Further trials are clearly needed extending the duration of CPAP treatment to the total duration of the weight loss programme and with adequate assessment of compliance and efficiency of CPAP treatment.

\section{Study strengths and limitations}

The primary outcome of this ancillary study was the changes in ODI after 1 year of lifestyle intervention in men with visceral obesity. Men having baseline ODI above or below 10 events/h were studied in post hoc analyses. The results have allowed us to identify the need for future studies that will address the response to lifestyle intervention of men with visceral obesity and untreated OSAS versus those of similar body weight and visceral fat without OSAS. Another limitation associated with this post hoc design is the imbalance between the two groups: 42 men having an ODI of less than 10 events/h and 24 having more than 10 events/h.

The particular strength of this study is the application of an integrated and synergistic lifestyle intervention programme which combined long-term moderate caloric restriction with an increase in moderate-intensity endurance exercise and occupational activity. This lifestyle modification programme was designed to be conducted in routine clinical practice, thus conclusions are extendable to the routine care of patients.

\section{CONCLUSION}

The findings of this study suggest that sleep-related breathing disorders are likely to impede the efficiency of a lifestyle intervention programme. Men with OSAS (ODI $\geq 10$ events/h) at baseline had a smaller reduction in body weight and smaller metabolic improvements associated with the lifestyle intervention programme than men without OSAS (ODI $<10$ events/h). 
Further studies should address whether CPAP treatment should be administered during the period of the lifestyle intervention programme to increase body weight loss and improve the cardiometabolic risk markers associated with such programmes in people with visceral obesity and OSAS.

Acknowledgements Dr Borel is a post-doctoral fellow supported by a fellowship from Agiràdom (Meylan, France) and the Rhône-Alpes region (France). Dr Paul Poirier is a senior clinical researcher of the Fonds de recherche en santé du Québec (FRSO).

Contributors All authors have substantially contributed to the study by participating in conception and design, or analysis and interpretation of data, drafting the paper or revising it critically for important intellectual content and have given their final approval of the version to be published. ALB: analysed data and wrote the paper. $\mathrm{XL}$ : contributed to data analysis; NA, AT, JB, PP, JPD, FS: researched data, contributed to discussion and reviewed/edited the paper.

Funding This study was supported by the Canadian Institutes of Health Research: CIHR grant 89985 and the National Institute for Public Research.

Competing interests None

Patient consent Obtained.

Ethics approval Ethics approval was provided by the Medical Ethics Committees of Université Laval and Institut universitaire de cardiologie et de pneumologie de Québec.

Provenance and peer review Not commissioned; externally peer reviewed.

\section{REFERENCES}

1. Despres JP, Lemieux I. Abdominal obesity and metabolic syndrome. Nature 2006:444:881-7

2. Marin JM, Carrizo SJ, Vicente E, et al. Long-term cardiovascular outcomes in men with obstructive sleep apnoea-hypopnoea with or without treatment with continuous positive airway pressure: an observational study. Lancet 2005;365:1046-53.

3. Despres JP. The insulin resistance-dyslipidemic syndrome of visceral obesity: effect on patients' risk. Obes Res 1998;6(Suppl 1):8S-17.

4. Rheaume C, Arsenault BJ, Belanger S, et al. Low cardiorespiratory fitness levels and elevated blood pressure: what is the contribution of visceral adiposity? Hypertension 2009;54:91-7.

5. Peppard PE, Young T, Palta M, et al. Prospective study of the association between sleep-disordered breathing and hypertension. N Engl J Med 2000;342:1378-84.

6. Vgontzas AN. Does obesity play a major role in the pathogenesis of sleep apnoea and its associated manifestations via inflammation, visceral adiposity, and insulin resistance? Arch Physiol Biochem 2008;114:211-23.

7. Romero-Corral A, Caples SM, Lopez-Jimenez F, et al. Interactions between obesity and obstructive sleep apnea: implications for treatment. Chest 2010;137:711-19.

8. Peppard PE, Young T, Palta M, et al. Longitudinal study of moderate weight change and sleep-disordered breathing. JAMA 2000;284:3015-21.

9. Peppard PE, Young T. Exercise and sleep-disordered breathing: an association independent of body habitus. Sleep 2004:27:480-4.

10. Chin K, Shimizu K, Nakamura T, et al. Changes in intra-abdominal visceral fat and serum leptin levels in patients with obstructive sleep apnea syndrome following nasal continuous positive airway pressure therapy. Circulation 1999:100:706-12.

11. Tuomilehto HP, Seppa JM, Partinen MM, et al. Lifestyle intervention with weight reduction: first-line treatment in mild obstructive sleep apnea. Am J Respir Crit Care Med 2009;179:320-7.

12. Foster GD, Borradaile KE, Sanders MH, et al. A randomized study on the effect of weight loss on obstructive sleep apnea among obese patients with type 2 diabetes: the Sleep AHEAD study. Arch Intern Med 2009;169:1619-26.

13. Johansson K, Neovius M, Lagerros YT, et al. Effect of a very low energy diet on moderate and severe obstructive sleep apnoea in obese men: a randomised controlled trial. BMJ 2009;339:b4609.
14. Tuomilehto $\mathbf{H}$, Gylling $\mathbf{H}$, Peltonen $\mathbf{M}$, et al. Sustained improvement in mild obstructive sleep apnea after a diet- and physical activity-based lifestyle intervention: postinterventional follow-up. Am J Clin Nutr 2010;92:688-96.

15. Johansson $\mathbf{K}$, Hemmingsson $E$, Harlid $R$, et al. Longer term effects of very low energy diet on obstructive sleep apnoea in cohort derived from randomised controlled trial: prospective observational follow-up study. BMJ 2011:342:d3017.

16. Medecine ACos. ACSM's Guidelines for Exercise Testing and Prescription. 5th edn Ney York, NY: Barnes and Noble, 1995.

17. Pare A, Dumont M, Lemieux I, et al. Is the relationship between adipose tissue and waist girth altered by weight loss in obese men? Obes Res 2001;9:526-34.

18. Ferland M, Despres JP, Tremblay A, et al. Assessment of adipose tissue distribution by computed axial tomography in obese women: association with body density and anthropometric measurements. Br J Nutr 1989;61:139-48.

19. Despres JP, Ross R, Boka G, et al. Effect of rimonabant on the high-triglyceride/lowHDL-cholesterol dyslipidemia, intraabdominal adiposity, and liver fat: the ADAGIOLipids trial. Arterioscler Thromb Vasc Biol 2009;29:416-23.

20. Arzt M, Young T, Finn L, et al. Sleepiness and sleep in patients with both systolic heart failure and obstructive sleep apnea. Arch Intern Med 2006;166:1716-22.

21. Vazquez JC, Tsai WH, Flemons WW, et al. Automated analysis of digital oximetry in the diagnosis of obstructive sleep apnoea. Thorax 2000:55:302-7.

22. Series F, Kimoff RJ, Morrison D, et al. Prospective evaluation of nocturnal oximetry for detection of sleep-related breathing disturbances in patients with chronic heart failure. Chest 2005:127:1507-14.

23. Randerath WJ, Verbraecken J, Andreas S, et al. Non-CPAP therapies in obstructive sleep apnoea. Eur Respir J 2011;37:1000-28.

24. Young T, Skatrud J, Peppard PE. Risk factors for obstructive sleep apnea in adults. JAMA 2004;291:2013-16

25. Kajaste S, Brander PE, Telakivi T, et al. A cognitive-behavioral weight reduction program in the treatment of obstructive sleep apnea syndrome with or without initial nasal CPAP: a randomized study. Sleep Med 2004;5:125-31.

26. Phillips BG, Hisel TM, Kato M, et al. Recent weight gain in patients with newly diagnosed obstructive sleep apnea. J Hypertens 1999;17:1297-300.

27. Traviss KA, Barr Sl, Fleming JA, et al. Lifestyle-related weight gain in obese men with newly diagnosed obstructive sleep apnea. J Am Diet Assoc 2002:102:703-6.

28. Sharma SK, Agrawal S, Damodaran D, et al. CPAP for the metabolic syndrome in patients with obstructive sleep apnea. N Engl J Med 2011;365:2277-86.

29. Spiegel K, Leproult R, Van Cauter E. Impact of sleep debt on metabolic and endocrine function. Lancet 1999;354:1435-9.

30. Spiegel K, Tasali E, Penev $P$, et al. Brief communication: sleep curtailment in healthy young men is associated with decreased leptin levels, elevated ghrelin levels, and increased hunger and appetite. Ann Intern Med 2004;141:846-50.

31. Nedeltcheva AV, Kilkus JM, Imperial J, et al. Insufficient sleep undermines dietary efforts to reduce adiposity. Ann Intern Med 2010;153:435-41.

32. Chaput JP, Leblanc C, Perusse L, et al. Risk factors for adult overweight and obesity in the Quebec Family Study: have we been barking up the wrong tree? Obesity /Silver Spring) 2009;17:1964-70.

33. Van Cauter E, Knutson KL. Sleep and the epidemic of obesity in children and adults Eur J Endocrinol 2008;159(Suppl 1):S59-66.

34. Stamatakis KA, Punjabi NM. Effects of sleep fragmentation on glucose metabolism in normal subjects. Chest 2010;137:95-101.

35. Tasali E, Leproult R, Ehrmann DA, et al. Slow-wave sleep and the risk of type 2 diabetes in humans. Proc Natl Acad Sci U S A 2008;105:1044-9.

36. Carneiro G, Togeiro SM, Hayashi LF, et al. Effect of continuous positive airway pressure therapy on hypothalamic-pituitary-adrenal axis function and 24-h blood pressure profile in obese men with obstructive sleep apnea syndrome. Am J Physiol Endocrinol Metab 2008;295:E380-4.

37. Major GC, Series F, Tremblay A. Does the energy expenditure status in obstructive sleep apnea favour a positive energy balance? Clin Invest Med 2007;30: E262-8

38. Hins J, Series F, Almeras N, et al. Relationship between severity of nocturnal desaturation and adaptive thermogenesis: preliminary data of apneic patients tested in a whole-body indirect calorimetry chamber. Int J Obes (Lond) 2006;30:574-7.

39. Stenlof K, Grunstein R, Hedner J, et al. Energy expenditure in obstructive sleep apnea: effects of treatment with continuous positive airway pressure. Am J Physiol 1996;271:E1036-43 